\title{
Verification of SGS-Stress Models by Direct Numerical Simulation of Compressible Homogeneous Isotropic Turbulence*
}

\author{
Toshio MIYAUCHI**, Mamoru TANAHASHI** \\ and Kunihiko MIYAKE**
}

\begin{abstract}
Direct numerical simulation of three-dimensional compressible homogeneous isotropic turbulence was conducted by a spectral method to verify SGS models of large eddy simulation. The results obtained using a linear combination model and dynamic eddy viscosity model were compared with the exact SGS stress evaluated from the DNS data. The following conclusions are obtained. (1) Correlation coefficients between the exact cross term and the one predicted using the linear combination model and the original scale similarity hypothesis show values as high as $90 \%$. (2) The SGS Reynolds stress predicted using the assumption of scale similarity shows the highest correlation with the filtered DNS data, while the correlation between the exact value and the value predicted using the linear combination model is low. (3) The stress predicted using the dynamic SGS model and the exact stress show low correlation which depends on the filter selection and the averaging volume.
\end{abstract}

Key Words: Computational Fluid Dynamics, Numerical Analysis, Turbulence, Compressible Flow, Direct Numerical Simulation, Large Eddy Simulation, SGS Model, Spectral Method

\section{Introduction}

With the recent advancement of computer technology, numerical simulations have become as important as theoretical and experimental study in turbulence research. However, the complicated nature of compressible turbulence prevents rapid progress in its study by means of numerical simulation.

The large eddy simulation (LES) of turbulent flows possesses great potential for numerically predicting turbulent flows. In LES, flow is divided into grid scale and subgrid scale by a filter. As a result of this decomposition, subgrid-scale stress terms appear in the governing equations and need to be modeled to close the equations. For compressible turbulence, Yoshizawa ${ }^{(1)}$ proposed an eddy viscosity model. Erlebacher and $\mathrm{co}^{-}$workers ${ }^{(2),(3)}$ proposed a linear combination model based on scale similarity ${ }^{(4)}$ and an eddy

* Received 7th August, 1995. Japanese original: Trans. Jpn. Soc. Mech. Eng., Vol.61, No.582, B (1995), pp. 413-419 (Received 4th April, 1994)

** Department of Mechano-Aerospace Engineering, Tokyo Institute of Technology, 2-12-1 Ookayama, Meguro-ku, Tokyo 152, Japan viscosity model. Moin et al. ${ }^{(5)}$ also proposed a dynamic eddy viscosity model for compressible turbulence. However, a practical and efficient compressible SGS model is not yet available. In this work, assessment of SGS models for compressible turbulent flows based on the DNS data base of compressible homogeneous isotropic turbulence is conducted.

Governing equations and SGS models for compressible turbulence are described in section 3 and DNS results of compressible homogeneous isotropic turbulence are presented in section 4 . Compressible SGS models are verified in section 5 . Conclusions of this study are summarized in section 6 .

\section{Nomenclature}

$\rho:$ Nondimensional density

$u_{i}$ : Nondimensional velocity

$p$ : Nondimensional pressure

$T$ : Nondimensional temperature

$\gamma:$ Specific heat ratio $\left(\mathrm{c}_{p} / \mathrm{c}_{v}\right)$

$R e$ : Reynolds number

Pr: Prandtl number

$M_{0}$ : Turbulent Mach number

$E$ : Kinetic energy 


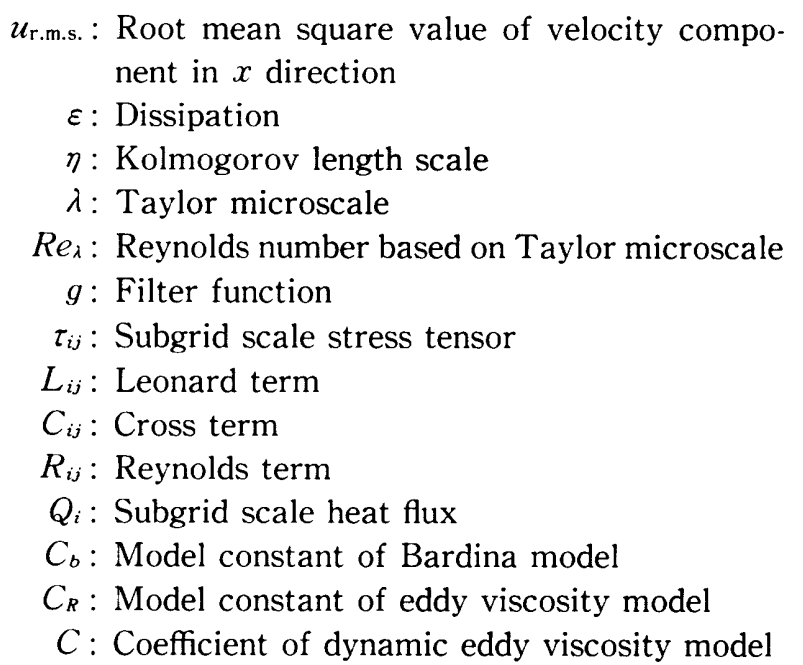

Symbols

- : Grid-scale variable

: Grid-scale variable (Favre average)

$'$ : Subgrid-scale variable

$\langle>$ : Spatial average

\section{Governing Equations and SGS Models for Compressible Turbulence}

\section{1 Governing equations for compressible flow}

In this work, it is assumed that external forces, bulk viscosity and radiative heat transfer are negligible, and the fluid obeys the equation of state for a perfect gas. With the above assumptions, the governing equations for compressible flows can be derived as follows ;

$$
\begin{aligned}
& \frac{\partial \rho}{\partial t}+\frac{\partial \rho u_{i}}{\partial x_{i}}=0 \\
& \frac{\partial \rho u_{i}}{\partial t}+\frac{\partial \rho u_{i} u_{j}}{\partial x_{j}}=-\frac{\partial p}{\partial x_{i}}+\frac{\partial \sigma_{i j}}{\partial x_{j}}, \\
& \frac{\partial p}{\partial t}+u_{i} \frac{\partial p}{\partial x_{i}}+\gamma p \frac{\partial u_{i}}{\partial x_{i}}=\frac{1}{\operatorname{PrM}_{0}^{2} \operatorname{Re}} \frac{\partial^{2} T}{\partial x_{i} \partial x_{i}}+(\gamma-1) \Phi
\end{aligned}
$$$$
p=\frac{\rho T}{\gamma M_{0}^{2}},
$$

where these equations are non-dimensionalized by the initial r.m.s. velocity $U_{0}, 1 / 2 \pi$ of the length of a computational domain $L_{0}$, initial mean density $\rho_{0}$ and initial mean temperature $T_{0}$. The conservation equation of energy (Eq. ( 3 )) is represented in terms of the pressure. Viscous stress tensor $\sigma_{i j}$ and viscous dissipation $\Phi$ are defined as follows ;

$$
\begin{aligned}
& \sigma_{i j}=-\frac{2}{3} \frac{1}{\operatorname{Re}} \frac{\partial u_{k}}{\partial x_{k}} \delta_{i j}+\frac{1}{\operatorname{Re}}\left(\frac{\partial u_{i}}{\partial x_{j}}+\frac{\partial u_{j}}{\partial x_{i}}\right) \\
& \Phi=-\frac{2}{3} \frac{1}{\operatorname{Re}}\left(\frac{\partial u_{k}}{\partial x_{k}}\right)^{2}+\frac{1}{\operatorname{Re}}\left(\frac{\partial u_{i}}{\partial x_{j}}+\frac{\partial u_{j}}{\partial x_{i}}\right) \frac{\partial u_{i}}{\partial x_{j}}
\end{aligned}
$$

Here, we define the following filter based on the Favre average ;

$$
f=\tilde{f}+f^{\prime},
$$

$$
\begin{aligned}
& \tilde{f}=\frac{\overline{\rho f}}{\bar{\rho}}, \\
& \bar{f}(x)=\int_{-\infty}^{\infty} g\left(x-x^{\prime}\right) f\left(x^{\prime}\right) d x^{\prime} .
\end{aligned}
$$

Applying the Favre averaged filter to the above governing equations, one can obtain the following equations for grid scale variables.

$$
\begin{aligned}
& \frac{\partial \bar{\rho}}{\partial t}+\frac{\partial \bar{\rho} \tilde{u}_{i}}{\partial x_{i}}=0 \\
& \frac{\partial \bar{\rho} \tilde{u}_{i}}{\partial t}+\frac{\partial \bar{\rho} \tilde{u}_{i} \tilde{u}_{j}}{\partial x_{j}}=-\frac{\partial \bar{p}}{\partial x_{i}}+\frac{\partial \bar{\sigma}_{i j}}{\partial x_{j}}-\frac{\partial \tau_{i j}}{\partial x_{j}}, \\
& \frac{\partial \bar{p}}{\partial t}+\tilde{u}_{i} \frac{\partial \bar{p}}{\partial x_{i}}+\gamma \bar{p} \frac{\partial \tilde{u}_{i}}{\partial x_{i}}=\frac{1}{\operatorname{PrM}_{0}^{2} \operatorname{Re}} \frac{\partial^{2} \tilde{T}}{\partial x_{i} \partial x_{i}}+(\gamma-1) \bar{\Phi} \\
& \quad-\frac{1}{M_{0}^{2}} \frac{\partial Q_{i}}{\partial x_{i}} \\
& \bar{p}=\frac{\bar{\rho} \tilde{T}}{\gamma M_{0}^{2}}
\end{aligned}
$$

where SGS stress tensor $\tau_{i j}$ and SGS heat flux $Q_{i}$ are represented as follows ;

$$
\begin{aligned}
& \tau_{i j}=L_{i j}+C_{i j}+R_{i j}, \\
& Q_{i}=\bar{\rho}\left(\tilde{u}_{i} \tilde{T}-\tilde{u}_{i} \tilde{T}+\widetilde{u_{i} T^{\prime}}+\widetilde{u_{i}^{\prime} \tilde{T}}+\widetilde{u_{i}^{\prime} T^{\prime}}\right) .
\end{aligned}
$$

In Eq. (14), $L_{i j}, C_{i j}$ and $R_{i j}$ are called the Leonard term, the cross term and the Reynolds term respectively and defined as follows ;

$$
\begin{aligned}
& L_{i j}=\bar{\rho}\left(\tilde{\tilde{u}}_{i} \tilde{u}_{j}-\tilde{u}_{i} \tilde{u}_{j}\right), \\
& C_{i j}=\bar{\rho}\left(\tilde{u}_{i}^{\prime} \tilde{u}_{j}+\tilde{u}_{i} u_{j}^{\prime}\right), \\
& R_{i j}=\bar{\rho} u_{i}^{\prime} u_{j}^{\prime} .
\end{aligned}
$$

In this study, SGS stress $\tau_{i j}$ is examined for compressible homogeneous isotropic turbulence.

\section{2 SGS models for compressible turbulence}

The Leonard term $L_{i j}$ in Eq. (14) can be directly calculated using the filter. However, the cross term $C_{i j}$ and the Reynolds term $R_{i j}$ must be modeled using the grid scale variables to conduct LES. Erlebacher et al. ${ }^{(2)}$ and Speziale et al..$^{(3)}$ proposed the following linear combination model which consists of an eddy viscosity model for the Reynolds term and the Bardina model for the cross term ;

$$
\begin{aligned}
& \tau_{i j}-\frac{1}{3} \bar{\rho} \widetilde{u_{m}^{\prime} u_{m}^{\prime} \delta_{i j}}=\bar{\rho}\left(\widetilde{\tilde{u}_{i} \tilde{u}_{j}}-\tilde{u}_{i} \tilde{u}_{j}\right) \\
& +\underbrace{\bar{\rho}\left(\tilde{u}_{i} \tilde{u}_{j}-\tilde{u}_{i} \tilde{u}_{j}\right)}_{C_{i j}}-\underbrace{2 C_{R} \bar{\rho} \Delta^{2}|\tilde{S}|\left(\tilde{S}_{i j}-\frac{1}{3} \tilde{S}_{m m} \delta_{i j}\right)}_{R_{i j}},
\end{aligned}
$$

where

$$
\widetilde{S}_{i j}=\frac{1}{2}\left(\frac{\partial \tilde{u}_{i}}{\partial x_{j}}+\frac{\partial \tilde{u}_{j}}{\partial x_{i}}\right) .
$$

In the above model, however, the cross term is different from that corresponding to the original scale similarity hypothesis ${ }^{(4)}$. Following the original scale similarity hypothesis, the cross term should be represented as follows;

$$
\begin{aligned}
& u_{i}^{\prime} \tilde{u}_{j} \cong \tilde{u}_{i}^{\prime} \tilde{u}_{j}=\left(\tilde{u}_{i}-\tilde{u}_{i}\right) \tilde{\tilde{u}}_{j}, \\
& \tilde{u}_{i} u_{j}^{\prime} \cong \tilde{u}_{i} u_{j}^{\prime}=\tilde{u}_{i}\left(\tilde{u}_{j}-\tilde{u}_{j}\right) .
\end{aligned}
$$

Furthermore, the Reynolds term should be modeled as 
follows ;

$$
u_{i}^{\prime} u_{j}^{\prime} \cong \tilde{u}_{i}^{\prime} \tilde{u}_{j}^{\prime}=\left(\tilde{u}_{i}-\tilde{u}_{i}\right)\left(\tilde{u}_{j}-\tilde{u}_{j}\right)
$$

If one uses these representations, the cross term of the linear combination model in Eq. (19) is given as the sum of Eqs. (21),(22) and (23). Thus the cross term in the linear combination model includes the Reynolds stress term. By considering the form invariant under the Galilean transformation, it is found that coefficients of the cross terms represented by Eqs. (21) and (22) must be $1^{(6)}$. However that of the Reynolds term (Eq. (23)) cannot be determined from the Galilean invariance.

In this study, the following model, which is based on the Bardina model according to the original scale similarity hypothesis, is considered;

$$
\tau_{i j}=\bar{\rho}\left(\tilde{u}_{i} \tilde{u}_{j}-\tilde{u}_{i} \tilde{u}_{j}\right)+\underbrace{\bar{\rho}\left\{\left(\tilde{u}_{i}-\tilde{u}_{i}\right) \tilde{u}_{j}+\tilde{u}_{i}\left(\tilde{u}_{j}-\tilde{u}_{j}\right)\right\}}_{C_{i j}}+\underbrace{C_{B} \bar{\rho}\left(\tilde{u}_{i}-\tilde{u}_{i}\right)\left(\tilde{u}_{j}-\tilde{\tilde{u}}_{j}\right.}_{R_{i j}})
$$

Hereafter the above model is referred to as Model 1. A modified linear combination model (Model 2) is also examined. Model 2 is constructed by adding an eddy viscosity model to Model 1, which can be represented as follows :

$$
\begin{aligned}
& \tau_{i j}-\frac{1}{3} \bar{\rho} \widetilde{u}_{m}^{\prime} u_{m}^{\prime} \delta_{i j}=\bar{\rho}\left(\widetilde{u}_{i} \tilde{u}_{j}-\tilde{u}_{i} \tilde{u}_{j}\right)+\underbrace{\bar{\rho}\left\{\left(\bar{u}_{i}-\tilde{u}_{i}\right) \tilde{u}_{j}+\tilde{u}_{i}\left(\tilde{u}_{j}-\tilde{u}_{j}\right)\right.}_{C_{i j}}\} \\
& \left.+C_{B} \bar{\rho}\left(\tilde{u}_{i}-\tilde{\tilde{u}}_{i}\right)\left(\tilde{u}_{j}-\tilde{u}_{j}\right)-2 C_{R} \bar{\rho} \Delta^{2}|\tilde{S}|\left(\tilde{S}_{i j}-\frac{1}{3} \tilde{S}_{m m} \delta_{i j}\right)\right) .
\end{aligned}
$$

Moin et al. ${ }^{(5)}$ extended the dynamic eddy viscosity model to compressible turbulence. In the dynamic model, the total SGS stress is represented by the following equation;

$$
\left.\tau_{i j}-\frac{1}{3} \tau_{m m} \delta_{i j}=-2 C \bar{\rho} \Delta^{2} \mid \tilde{S}\right)\left(\tilde{S}_{i j}-\frac{1}{3} \tilde{S}_{m m} \delta_{i j}\right),
$$

where coefficient $C$ is determined dynamically by applying the test filter ;

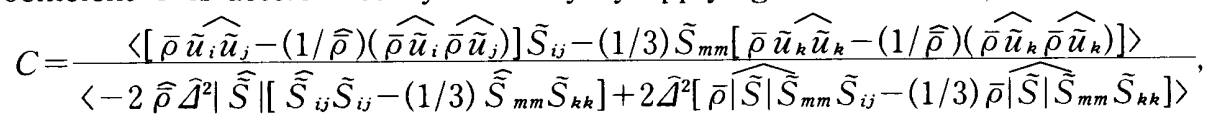

where $\langle>$ represents a spatial average. In general, the average is defined by a plane parallel to the wall in turbulent channel flow and the whole computational domain in isotropic turbulence. However, in the case of complex geometry, the average should be taken in a local volume. Therefore in this work, two volumes are used to take an average. One is the whole computational domain and the other is a cube with an integral length scale. The Gaussian filter and the sharp cut-off filter are used to clarify the effect of the filter.

\section{DNS of Compressible Homogeneous Isotropic Turbulence}

The DNS data which is used for assessment of SGS models is obtained by solving Eqs. (1)-(4) directly using a spectral method. Aliasing error is fully removed using the $3 / 2$ rule. A periodic boundary condition is applied in three directions and the secondorder Adams-Bashforth scheme is used for time integration. For the initial velocity field, a random field which has the same energy spectrum profile as the experimental result of grid turbulence obtained by Comte-Bellot and Corrsin ${ }^{(7)}$ is used. The initial density and pressure fluctuations are set equal to zero. The calculation is conducted for $R e=230.2, M_{0}=0.2$,
$0.4, \operatorname{Pr}=0.7$ and $\gamma=1.4$ on $128 \times 128 \times 128$ grids.

Table 1 Experimental results obtained by Comte-Bellot \& Corrsin and initial conditions for DNS of compressible homogeneous isotropic turbulence

\begin{tabular}{|c|c|c|}
\hline & CB \& C & Initial condition \\
\hline$E$ & 1.50 & 1.50 \\
\hline$u_{\text {r.m.s. }}$ & 1.00 & 1.00 \\
\hline$\varepsilon$ & 2.25 & 2.18 \\
\hline$\eta$ & 0.0145 & 0.0139 \\
\hline$\lambda$ & 0.177 & 0.173 \\
\hline$R e_{\lambda}$ & 38.1 & 39.8 \\
\hline
\end{tabular}

Table 2 Results of DNS at $t=0.5206$ and the corresponding experimental data obtained by Comte-Bellot \& Corrsin

\begin{tabular}{|c|c|c|c|}
\hline & CB \& C & $M_{0}=0.2$ & $M_{0}=0.4$ \\
\hline$E$ & 0.833 & 0.838 & 0.823 \\
\hline$u_{\text {r.m.s. }}$ & 0.745 & 0.749 & 0.740 \\
\hline$\varepsilon$ & 0.753 & 0.789 & 0.741 \\
\hline$\eta$ & 0.0191 & 0.0180 & 0.0182 \\
\hline$\lambda$ & 0.228 & 0.216 & 0.213 \\
\hline$R e_{\lambda}$ & 36.6 & 37.1 & 36.3 \\
\hline
\end{tabular}




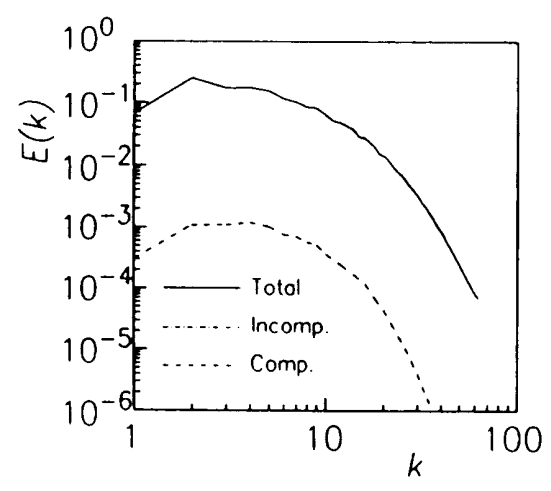

(a) $\quad M_{0}=0.2$

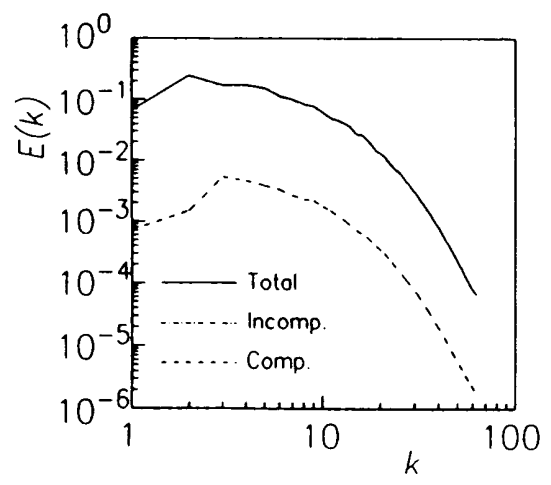

(b) $\quad M_{0}=0.4$

Fig. 1 Energy spectrum at $t=0.5206$

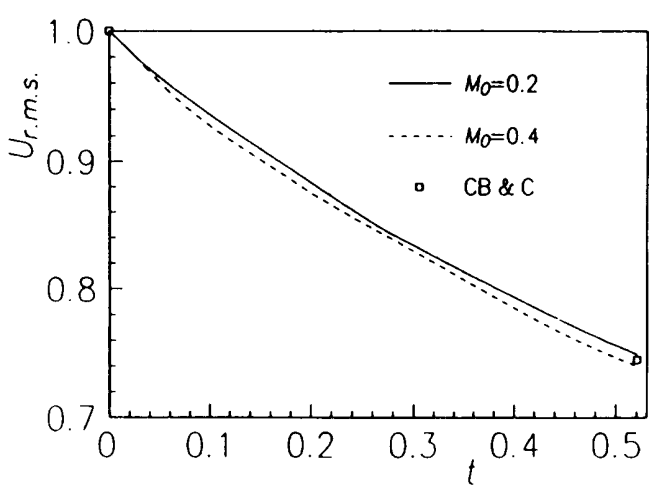

Fig. 2 Temporal development of r.m.s. velocity in the $x$ direction

The experimental results obtained by ComteBellot and Corrsin ${ }^{(7)}$ and the numerically obtained initial condition are shown in Table 1. It can be seen that the numerical initial condition coincides with the experimental data. Table 2 shows the numerical results at $t=0.5206$ and corresponding experimental results. The present result and the experimental one show good agreement. Figure 1 shows energy spectra at $t=0.5206$. Here, the solid line represents total energy. Dotted lines and dashed lines denote an incompressible component ( $\operatorname{div} \boldsymbol{u}=0$ ) and a compressible component (rot $\boldsymbol{u}=0)$, respectively. The two

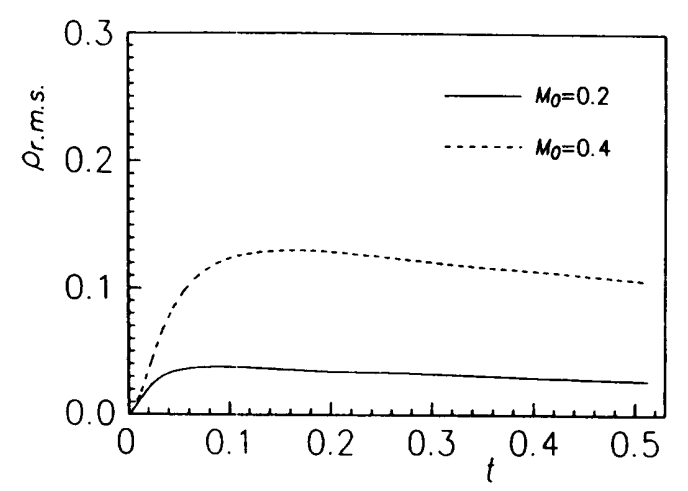

Fig. 3 Temporal development of density fluctuation

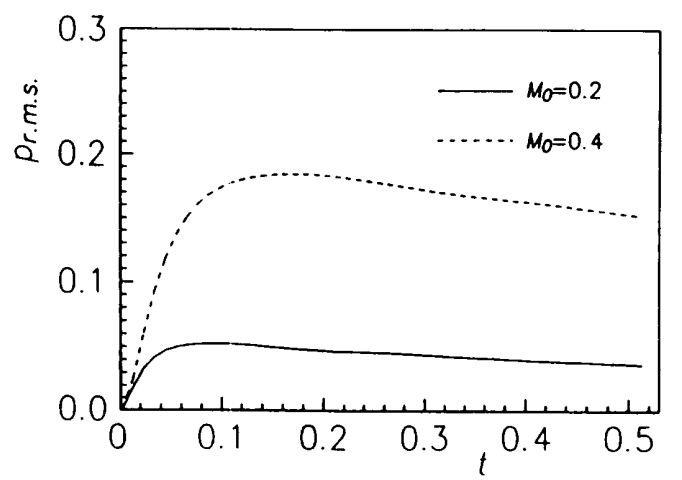

Fig. 4 Temporal development of pressure fluctuation

parts are calculated by Helmholtz decomposition. The compressible component of turbulent energy is $0.54 \%$ for $M_{0}=0.2$ and $2.3 \%$ for $M_{0}=0.4$ at $t=0.5206$. The decay of r.m.s. velocity fluctuation in the $x$ direction is shown in Fig. 2. The r.m.s. value of the velocity fluctuation decreases with time, and the decrease is faster for the larger Mach number. The results for two different Mach numbers show good agreement with the corresponding experimental results which are plotted in Fig. 2 using square symbols. Figures 3 and 4 show the temporal development of density and pressure fluctuations. Both fluctuations, which are initially zero, increase in the initial stage, reach maxima and decay gradually with time. As the Mach number increases, the fluctuations of density and pressure become larger.

\section{Assessment of SGS Models for Compressible Turbulence}

In this section DNS data of compressible homogeneous isotropic turbulence at $t=0.5206$ is used for assessment of SGS models for compressible turbulence. LES data on $32 \times 32 \times 32$ grids is obtained from DNS data on $128 \times 1.28 \times 128$ grids by filtering.

\section{1 Verification of the cross term}

In Table 3, correlation coefficients between DNS data and models for the cross term at $t=0.5206$ are 
shown for the case with Gaussian filter. Here the correlation coefficient is defined as follows ;

$$
C(f, g)=\frac{\langle f g\rangle}{\left\langle f^{2}\right\rangle^{\frac{1}{2}}\left\langle g^{2}\right\rangle^{\frac{1}{2}}} .
$$

It should be noted that the Bardina model which requires double filtering cannot be applied to the case with the sharp cut-off filter. Table 3 suggests that the linear combination model, Model 1 and Model 2 show very high correlation, as high as $90 \%$. These results imply that the scale similarity hypothesis is adequate. Moreover, the linear combination model, which includes Reynolds stress of the Bardina model in the cross term, shows high correlation. Mach number effect is not observed in the range of Mach number

Table 3 Correlation coefficient between DNS data and the models for the cross term

\begin{tabular}{|c|c|c|c|c|}
\hline \multirow{2}{*}{} & \multicolumn{2}{|c|}{ Linear combination } & \multicolumn{2}{c|}{ model 1 \& model 2 } \\
\cline { 2 - 5 } & $M_{0}=0.2$ & $M_{0}=0.4$ & $M_{0}=0.2$ & $M_{0}=0.4$ \\
\hline$(1,1)$ & 0.912 & 0.913 & 0.897 & 0.898 \\
\hline$(1,2)$ & 0.921 & 0.922 & 0.914 & 0.916 \\
\hline
\end{tabular}

Table 4 Ratios of r.m.s. values of the cross terms of DNS data and the models

\begin{tabular}{|c|c|c|c|c|}
\hline \multirow{2}{*}{} & \multicolumn{2}{|c|}{ Linear combination } & \multicolumn{2}{c|}{ model 1 \& model 2 } \\
\cline { 2 - 5 } & $M_{0}=0.2$ & $M_{0}=0.4$ & $M_{0}=0.2$ & $M_{0}=0.4$ \\
\hline$(1,1)$ & 1.280 & 1.277 & 1.431 & 1.429 \\
\hline$(1,2)$ & 1.056 & 1.055 & 1.162 & 1.161 \\
\hline
\end{tabular}

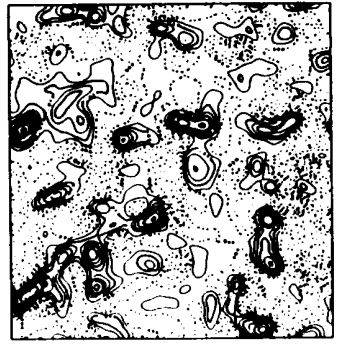

(a) DNS data

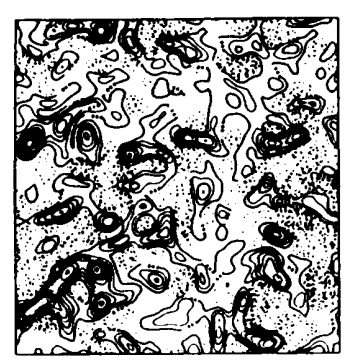

(b) Model $1 \&$ Model 2

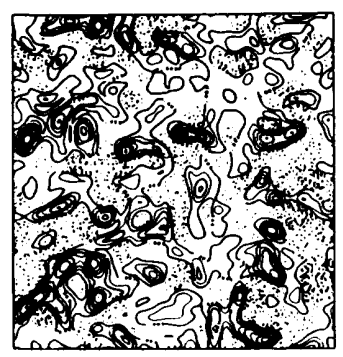

(c) Linear combination model

Fig. 5 Contour plots of the cross term $C_{12}\left(M_{0}=0.4\right)$ calculated in this study. Figure 5 shows contour plots of $C_{12}$ at $x=\pi$. These contours also suggest that the local structure of the cross term is accurately predict ed by the Bardina model. Table 4 shows the ratios of r.m.s. values of the cross terms of DNS data and the models. $C_{11}$ values calculated using the models are slightly larger than that of DNS data. However, the ratio is fairly close to 1 .

\section{2 Verification of the Reynolds term}

The correlation coefficients of the Reynolds stress obtained by applying the Gaussian filter are shown in Tables 5 and 6 for two different Mach numbers. Here it is assumed that the coefficients of scale similarity part $C_{b}$ and eddy viscosity part $C_{R}$ are equal in Model 2. Model 1, in which the Reynolds stress is simply

Table 5 Correlation coefficient between DNS data and the models for the Reynolds term in the case of $M_{0}=0.2$

\begin{tabular}{|c|c|c|c|}
\hline & Linear combination & model 1 & model 2 \\
\hline$(1,1)$ & 0.220 & 0.714 & 0.224 \\
\hline$(1,2)$ & 0.247 & 0.579 & 0.252 \\
\hline
\end{tabular}

Table 6 Correlation coefficient between DNS data and the models for the Reynolds term in the case of $M_{0}=0.4$

\begin{tabular}{|c|c|c|c|}
\hline & Linear combination & model 1 & model 2 \\
\hline$(1,1)$ & 0.211 & 0.710 & 0.216 \\
\hline$(1,2)$ & 0.238 & 0.582 & 0.243 \\
\hline
\end{tabular}

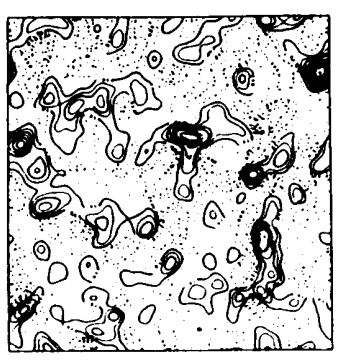

(a) DNS data

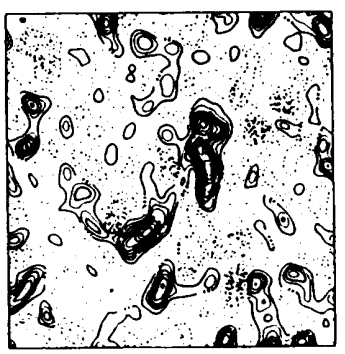

(c) Model 2

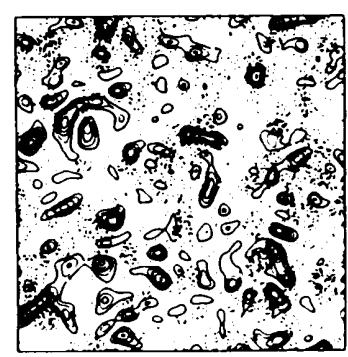

(b) Model 1

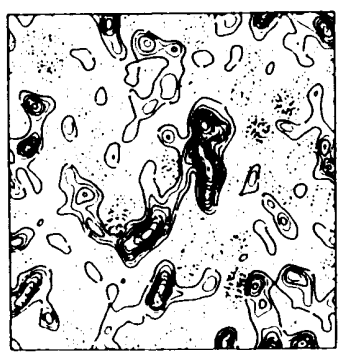

Fig. 6 Contour plots of the Reynolds stress $R_{12}\left(M_{0}=0.4\right)$ 
Fig. 7 Contour plots of the total SGS stress $\tau_{12}$ for dynamic eddy viscosity model (Gaussian filter, $M_{0}=0.4$ )

\begin{tabular}{|c|c|c|c|c|}
\hline \multirow{2}{*}{} & \multicolumn{2}{|c|}{ All domain } & \multicolumn{2}{c|}{ Integral scale } \\
\cline { 2 - 5 } & $M_{0}=0.2$ & $M_{0}=0.4$ & $M_{0}=0.2$ & $M_{0}=0.4$ \\
\hline$(1,1)$ & 0.297 & 0.283 & 0.280 & 0.274 \\
\hline$(1,2)$ & 0.328 & 0.323 & 0.312 & 0.303 \\
\hline
\end{tabular}

Table 8 Correlation coefficient between DNS data and the dynamic eddy viscosity model for $\tau_{i j}$ in the case of the sharp cut-off filter

\begin{tabular}{|c|c|c|c|c|}
\hline \multirow{2}{*}{} & \multicolumn{2}{|c|}{ All domain } & \multicolumn{2}{c|}{ Integral scale } \\
\cline { 2 - 5 } & $M_{0}=0.2$ & $M_{0}=0.4$ & $M_{0}=0.2$ & $M_{0}=0.4$ \\
\hline$(1,1)$ & 0.0867 & 0.0885 & 0.0825 & 0.835 \\
\hline$(1,2)$ & 0.112 & 0.106 & 0.104 & 0.0991 \\
\hline
\end{tabular}

Table 9 Constants of the dynamic eddy viscosity model C

\begin{tabular}{|c|c|c|}
\hline & $\bar{M}_{0}=0.2$ & $M_{0}=0.2$ \\
\hline Gaussian filter & 0.0299471 & 0.0277532 \\
\hline Cut-off filter & 0.0258260 & 0.0239682 \\
\hline
\end{tabular}

obtained using the scale similarity assumption, shows the highest correlation among the three models. The linear combination model shows the lowest correlation of about $20 \%$ for both $C_{11}$ and $C_{12}$. Model 2, which is a combination of the eddy viscosity model and Model 1, improves the correlation because of the scale similarity part. As reported for incompressible turbulence, absolute values of the scale similarity part and the eddy viscosity part are significantly different. In this case, the eddy viscosity part dominates the Reynolds stress term. Thus, it is necessary to determine the coefficients of the two parts $C_{b}$ and $C_{R}$ separately. Figure 6 shows contour plots of $R_{12}$. Model 1 gives reasonably accurate prediction of $R_{12}$, while the predicted $R_{\mathbf{1 2}}$ has smaller scale than the exact $R_{12}$. The linear combination model and Model 2 cannot be used to predict local structure of the Reynolds stress, which is expected from the correlation coefficients shown in Tables 5 and 6 .

\section{3 Verification of total SGS stress}

In Tables 7 and 8 , the correlation coefficients between exact $\tau_{i j}$ and those predicted using the dynamic SGS model are shown. Here, test filter width is set equal to 2 times the width of the grid filter. The correlation is about $30 \%$ for the Gaussian filter, $10 \%$ for the sharp cut-off filter. From the viewpoint of the correlation with DNS data, the dynamic model seems to be inaccuarate. Moreover, smaller averaging volume reduces correlation. This fact suggests that the

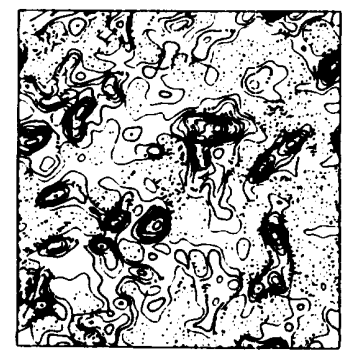

(a) DNS data

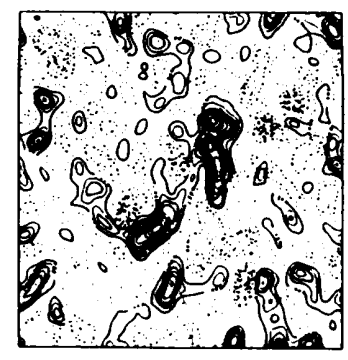

(b) Averaging volume is whole domain

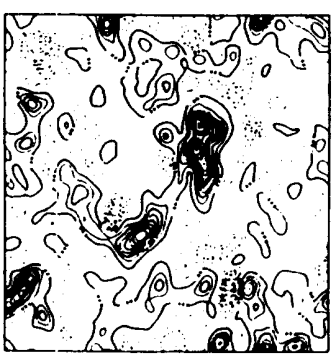

(c) Averaging volume is a cube of integral length

Table 7 Correlation coefficient between DNS data and the dynamic eddy viscosity model for $\tau_{i j}$ in the case of the Gaussian filter

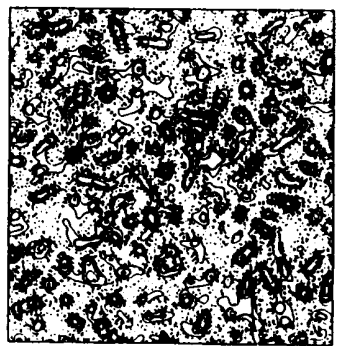

(a) DNS data

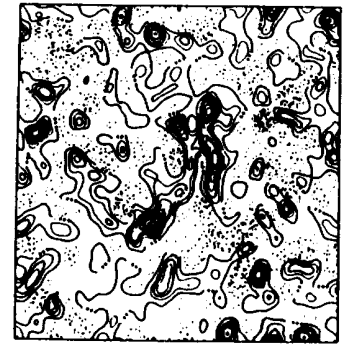

(b) Averaging volume is whole domain

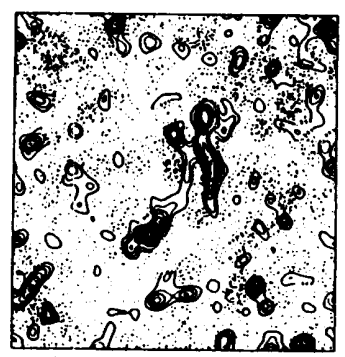

(c) Averaging volume is a cube of integral length

Fig. 8 Contour plots of the total SGS stress $\tau_{12}$ for dynamic eddy viscosity model (sharp cut ${ }^{-}$off filter, $\left.M_{0}=0.4\right)$

definition of an average volume is very important in calculation for the complex geometries. Table 9 shows the coefficient $C$. By taking a root, the 
corresponding Smagorinsky constant can be obtained. In general, the Smagorinsky constant is 0.2 for homogeneous isotropic turbulence. The constants calculated from Table 9 are $0.15-0.17$, which are consisitent with the above-mentioned value.

Figures 7 and 8 show contour plots of $\tau_{12}$ at $x=\pi$ for Gaussian and cut-off filters. The local structure of $\tau_{12}$ is not restored by the dynamic model. In Fig. 8, the exact $\tau_{12}$ calculated from DNS data using the cut-off filter shows very small-scale structure. For the Gaussian filter, subgrid scale components include large-scale energy, whereas those for the sharp cutoff filter consist of small scale energy below the cutoff wave number. As a result, the Gaussian filter yeilds higher correlation than that of the sharp cut-off filter.

\section{Conclusions}

In this study, SGS models for compressible turbulence were investigated using DNS data of compressible homogeneous isotropic turbulence. The models are verified in two flow fields with different Mach numbers. As a result, we have obtained the following conclusions.

(1) The exact cross term and the one predicted using the linear combination model and the original scale similarity hypothesis show very high correlation, as high as $90 \%$.

(2) The SGS Reynolds stress predicted using the scale similarity model shows the highest correlation with the filtered DNS data, whereas the correlation between the exact value and that predicted using the linear combination model is low.

(3) To improve the correlation of the SGS Reynolds stress term in model 2, coefficients of the eddy viscosity part and the scale similarity part must be determined independently.

(4) The correlation between the stress predicted using the dynamic SGS model and the exact stress is low. This correlation depends on the filter selection and the averaging volume.

\section{Acknowledgement}

This work was supported by the Japan Ministry of Education, Science and Culture under Grant No. 05240108 .

\section{References}

(1) Yoshizawa, A., Phys. Fluids, Vol. 29, No. 7(1986), p. 2152.

(2) Erlebacher, G., Speziale, C.G., Zang T.A. and Hussaini, M.Y., NASA CR-178273(1987).

( 3 ) Speziale, C.G., Erlebacher G., Zang T.A. and Hussaini, M.Y., Phys. Fluids, Vol. 31, No. 4(1988), p. 940.

(4) Bardina, J., Improved Turbulence Models Based on Large Eddy Simulation of Homogeneous, Incompressible Turbulent Flows, Ph. D. dissertation, Stanford Univ., (1983).

(5) Moin, P., Squires, W., Cabot and Lee, S., Phys. Fluids, A, Vol. 3, No.11(1991), p. 2746.

(6) Speziale, C.G., J. Fluid Mech., Vol. 156(1985), p. 55 .

( 7 ) Comte-Bellot, G. and Corrsin, S., J. Fluid Mech., Vol. 48(1971), p. 273. 